\title{
Utilization of Mobile Application for Better Implementation of Good Clinical Practice in a Biorepository Sample Collection Process: Functions of PancMoBio in Biobanking
}

\author{
Lei Tian, ${ }^{1-3,{ }^{*}}$ Kai Zhang, ${ }^{1-3,{ }^{*}}$ Xiao Wang, ${ }^{4}$ Kuirong Jiang, ${ }^{1-3}$ Wentao Gao, ${ }^{1-3}$ Ningxia Liang, ${ }^{5}$ \\ Xiuqin Wang, ${ }^{5}$ Yi Miao, ${ }^{1-3}$ and Zipeng $\mathrm{Lu}^{1-3}$
}

Preanalytical variables of biospecimens play a vital role in biobanking. Currently, there is a lack of a convenient and precise methods to document these variables. Paper documentation and computer-based Lab Information Management System software are the most common solutions, but both have clear disadvantages. An application named PancMoBio was newly developed in our pancreas biobank, with the guidance of good clinical practice principles as well as the incorporation of technical support from professional software companies. With portable electronic devices running this application, all data can be precisely collected in a synchronous manner during sample collection and processing. PancMoBio comprises two major modules-recording and searching - and five submodules in the recording module: blood sample collection, solid tumor tissue sample collection, cystic tumor sample collection, plasma sample separation, and serum sample separation. Compared with other methods, our application was found to be more convenient and accurate in recording preanalytical variables and demonstrated improved capability in facilitating real-time quality control and quality assurance. It was apparent that PancMoBio could improve the integrity of biospecimen and biobank quality management. Thus, it should be considered for further utilization in biobanking.

Keywords: good clinical practice, smartphone application, preanalytical variables, sample collection, biobanking

\section{Introduction}

$\mathrm{B}$ IOLOGICAL SAMPLES ARE important for scientific research. Biobanking involves multiple steps, such as collection, processing, transportation, storage, and retrieval of biospecimens. ${ }^{1}$ Biospecimens are a more ideal extended tool than cell lines for health and disease research, and can be used directly for metabonomics, genomics, transcriptomics, and proteomics analysis. High-quality biospecimens require appropriate management to ensure that they are reliable and stable. The integrity of biospecimens is fundamental to the provision of high-quality tissue samples for research. Preanalytical variables from the Standard Preanalytical Code (SPREC V2.0) developed by the International Society for Biological and Environmental Repositories (ISBER) comprise good practices, and can impact the integrity of bios- pecimens and their simple derivatives during collection, processing, and storage, including cold/warm ischemic time, centrifugation, and temperature. ${ }^{1,2}$ Good clinical practice (GCP) is an international quality standard that requires safety and privacy for donors, as well as traceability and reliability of data, and is a good reference standard for biobanking. ${ }^{3,4}$ Thus, the detailed information of these preanalytical variables plays a vital role in biobanking and is fundamental for research and interconnectivity and interoperability between national and international biobanks.*

There is, however, a lack of useful methods to ensure data collection, integrity, and reliability. Paper documentation and computer-based Lab Information Management

\footnotetext{
*http://www.isber.org/page/SPREC
}

\footnotetext{
${ }^{1}$ Pancreas Center and ${ }^{2}$ Pancreas Biobank, The First Affiliated Hospital of Nanjing Medical University, Nanjing, P.R. China.

${ }^{3}$ Pancreas Institute of Nanjing Medical University, Nanjing, P.R. China.

${ }^{4}$ Yozo Software Company, Wuxi, P.R. China.

${ }^{5}$ Department of Science and Technology, The First Affiliated Hospital of Nanjing Medical University, Nanjing, P.R. China.

*Contributed equally.
} 
System (LIMS) software is most commonly used in biobanking. Despite paper documentation being convenient to carry and record, it is susceptible to being revised or destroyed, whereas LIMS software tends to be troublesome in terms of mobility.

Applications for mobile smartphones and tablet devices are now becoming a major factor in daily life because mobile phone ownership is dramatically increasing globally. ${ }^{5}$ Internet-based and mobile applications have been widely developed to assist people with respect to monitoring, managing, and communicating multiple types of information, including that which is health related. ${ }^{6-9} \mathrm{~A}$ growing volume of examples of medical use of applications focus on medication, nursing, nutrition, blood pressure/ glucose monitoring, rehabilitation, and different kinds of diseases. ${ }^{10-14}$ However, to our knowledge, few applications have been developed for biobanking management. Therefore, a convenient and reliable application for recording all relevant data is vital and urgently required for the entire management process during sample collection and processing.

PancMoBio (Chinese version 1), an application for biobanking, was developed by the Pancreas Center of the First Affiliated Hospital of Nanjing Medical University. It contains two major modules-recording and searching-and five submodules in the recording module: blood sample collection, solid tumor sample collection, cystic tumor sample collection, plasma processing, and serum processing. The PancMoBio provides a convenient and efficient method for data recording for both sample collection and storage. In this article, the different functions of this application are discussed.

\section{Introduction of PancMoBio}

Our Pancreas Biobank was reviewed and approved by the Ethics Committee of the First Affiliated Hospital of Nanjing Medical University in accordance with the established standards of the handling of biospecimens. All participants completed unique consent forms. The PancMoBio (Chinese Version 1) was initiated in 2017, with the first Chinese version on the website of our Pancreas Center www .pancreas.org.cn/ being demonstrated in 2018. PancMoBio was developed for the IOS (Internetworking Operating System) platform and was a joint project with the biobank staff in our center and professional software engineers under the guidance of GCP principles. This application is currently only in Chinese, with PancMoBio (English Version 1) being released soon. The central feature of PancMoBio is data recording for the entire process of sample collection and processing, enabling biobankers to record operating information conveniently and in a timely manner. For this application, 31 variables with 4 main subcategories are described, including basic information (manipulator, device, reagent batch number, etc.), preanalytical variables (cold/warm ischemia time, etc.), quality control (QC, checkpoints in a standard operating procedure), and quality assurance (QA, automatic operation log recording) (Table 1).

\section{How to get started}

Before using this application, choosing an exclusive account, typing a login ID and password is a prerequisite, all of which can be used for access control permission. PancMoBio (Chinese Version 1) is divided into two major modules-recording and searching (Fig. 1). It is a simple matter to search for any patient by serial number in the search module. In the recording module, five submodules are provided: blood sample collection, solid tumor sample collection, cystic tumor sample collection, and blood sample processing (plasma and serum). The following provides further details regarding the use of these five submodules, and flowcharts can be seen in Figure 2. For getting to know how to use this APP clearly, see Figure 3.

\section{Blood collection}

Blood sample collection involves six steps after recording a new serial number for a sample through $\mathrm{QR}$ code scanning or manual input (Fig. 3).

(1) Record name of operator automatically according to login ID.

(2) Take photos of consent form.

(3) (I) Record current date and time automatically by pressing "Starting blood collection."

(II) Record name and employee number of the nurse who draws blood.

(4) Record whether blood collection tubes are treated according to standard operating procedures (SOPs).

Table 1 . Variables Recorded in PancMoBio

\begin{tabular}{|c|c|c|c|}
\hline Basic information & Preanalytical variables & Quality control & Quality assurance \\
\hline Serial number & Date and time of specimen collection & $\begin{array}{l}\text { Preprocessing of blood } \\
\text { collection tube (serum } \\
\text { tube, EDTA tube) }\end{array}$ & Photo of consent forms \\
\hline Specimen type & Time blood was delivered to biobank & Preprocessing of cystic fluid & Consent form version number \\
\hline \multirow[t]{4}{*}{ Volume of samples } & $\begin{array}{l}\text { Centrifugation (date and time, } \\
\text { device number, speed, } \\
\text { temperature, and duration) }\end{array}$ & $\begin{array}{l}\text { Transferring temperature } \\
\text { of cystic fluid }\end{array}$ & $\begin{array}{l}\text { Photo of specimen resected } \\
\text { from surgery }\end{array}$ \\
\hline & $\begin{array}{l}\text { Date and time that specimens } \\
\text { were put in freezer }\end{array}$ & \multirow[t]{3}{*}{$\begin{array}{l}\text { Whether processing time } \\
\text { coincides with standard } \\
\text { operating procedures }\end{array}$} & Special comments \\
\hline & Time of cutting off main blood supply & & Operator \\
\hline & Time of cutting off specimens & & $\begin{array}{l}\text { Nurse who drew blood } \\
\text { (name, employee number) } \\
\text { Attending surgeon }\end{array}$ \\
\hline
\end{tabular}




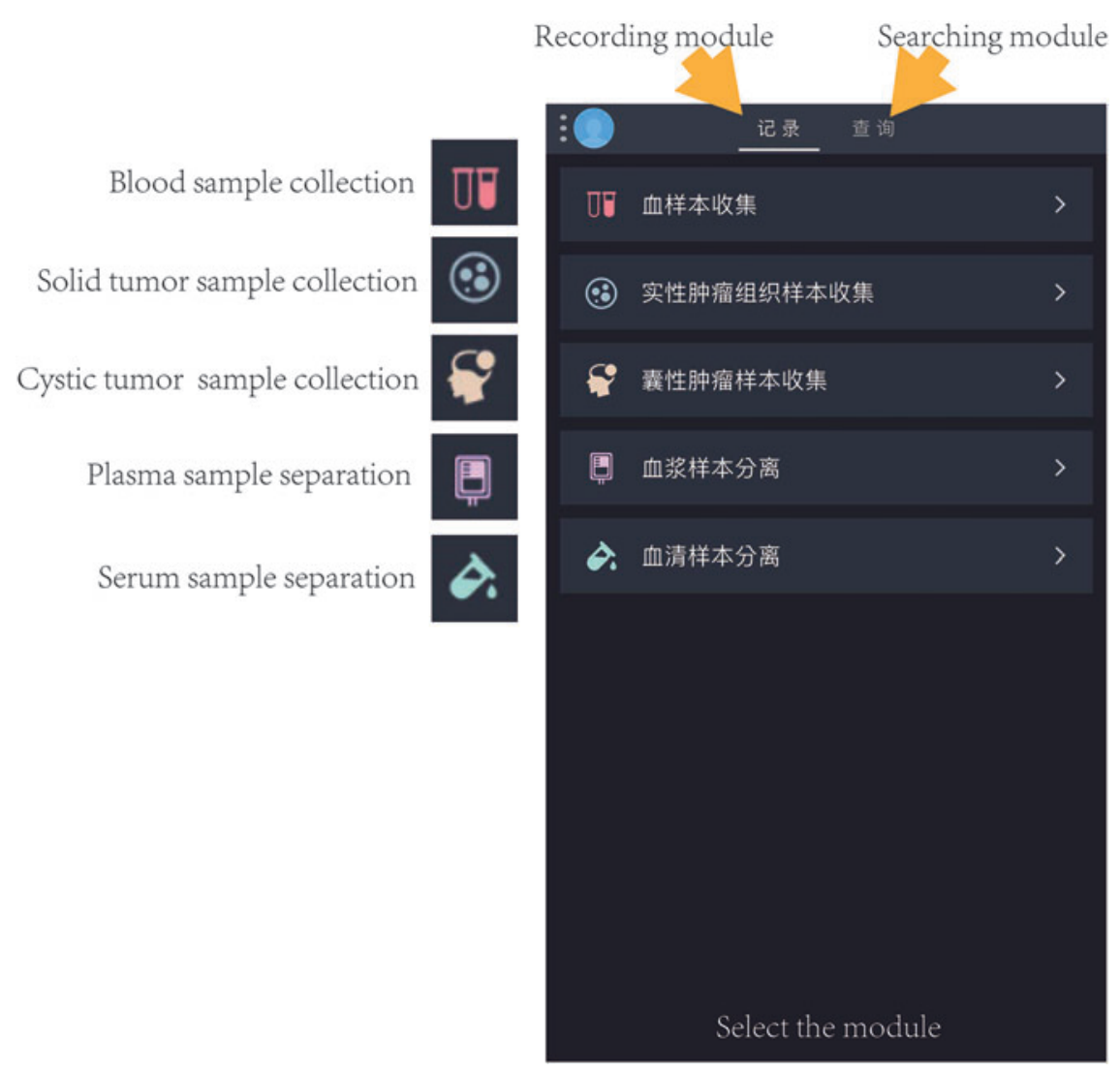

FIG. 1. The two major modulesrecording and searching and five submodules in recording module: blood collection, solid tumor collection, cystic tumor collection, plasma separation, and serum separation. Color images are available online.

\section{Blood sample collection \\ Solid tumor sample collection \\ Cystic tumor sample collection \\ Plasma sample separation \\ Serum sample separation}

血样本收隹
(5) Record current time automatically as samples are sent to biobank preprocessing room.

(6) Evaluate automatically whether time from blood collection to arrival at preprocessing room is $<40$ minutes

All data are then saved and uploaded to the local server.

\section{Plasma/serum separations}

After blood samples arrive at the preprocessing room, the technician who is in charge of sample processing will sign for them after confirming the sample identity by scanning the QR code or manual input. All of these blood samples will be sent to the "plasma/serum separation" process, which comprises eight stages (Fig. 3):

(1) Record name of operator automatically according to login ID.

(2) Record date and time of blood collection.

(3) For serum samples, confirm they are standing for 30 minutes before centrifugation. Skip this step for plasma samples.

(4) Record centrifugal condition details (speed, duration, temperature, number of device, date, and time).

(5) Record volume of every vial.

(6) Allocate location and record time when specimens are stored at $-80^{\circ} \mathrm{C}$.

(7) Evaluate automatically whether time from blood collection to storing at $-80^{\circ} \mathrm{C}$ is $<2$ hours.

(8) Record special comments if necessary.

All data are then saved and uploaded to the local server.

\section{Solid/cystic tumor collection}

When patients with informed consent are operated on, the technician will prepare the solid/cystic tumor sample collection kit, which contains paraffin embedding boxes for tumor tissues from a donor; for tissues adjacent to a tumor, the tubes contain these solid samples as well as cystic fluid. In the preparation room, the technician will deal with the samples and record the information for PancMoBio. The solid/cystic tumor sample collection involves 11 steps (Fig. 3):

(1) Record serial number and name of operator, as mentioned previously.

(2) Record date and time of sample collection.

(3) Record time of cutting main blood supplies and specimens.

(4) Record name of research fellow who offers information for this tumor sample.

(5) Take photos of specimens.

(6) For cystic tumor samples, record basic information of cystic fluid collection (numbers of tubes of cystic fluid, date and time of collection, whether cystic fluid is stored on ice); skip this step for solid tumor samples.

(7) Record time when tissue samples are put into liquid nitrogen or neutral buffered formalin.

(8) Evaluate automatically whether cold ischemia time is $<20$ minutes (coincided with SOPs).

(9) Allocate location and record time when specimens are stored.

(10) For cystic tumor samples, record whether cystic fluid is treated according to SOPs; skip this step for solid tumor samples. 


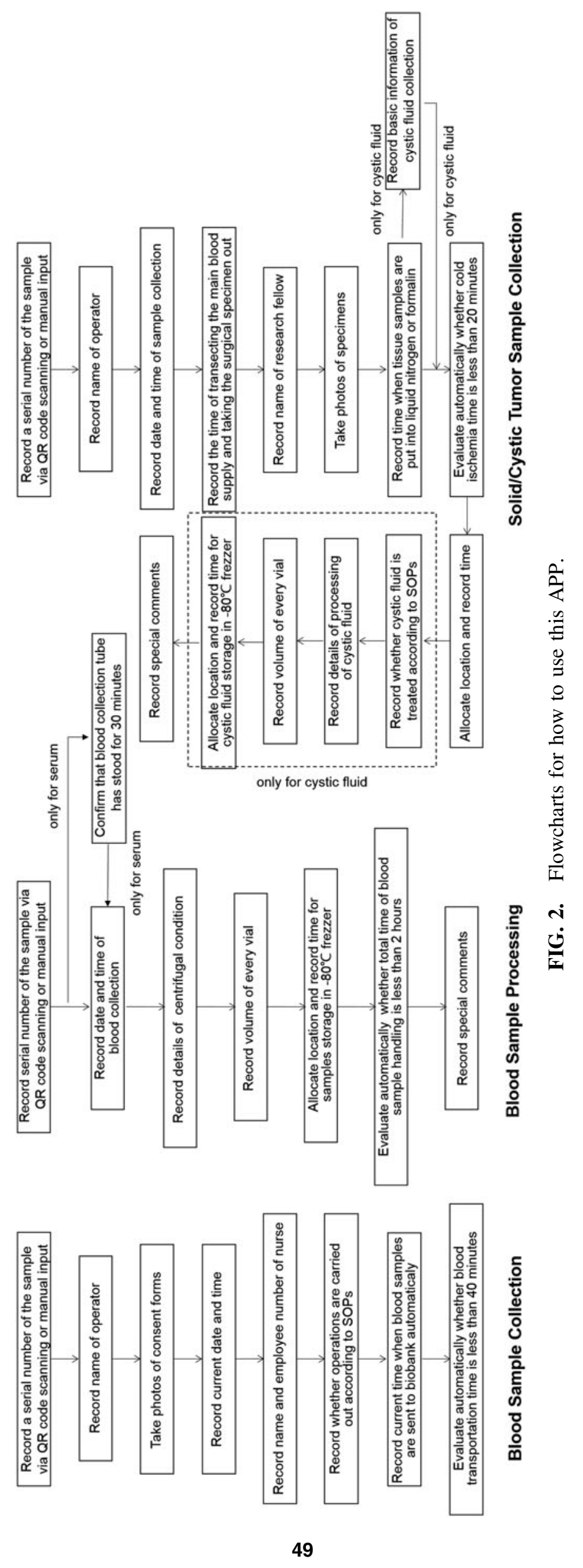




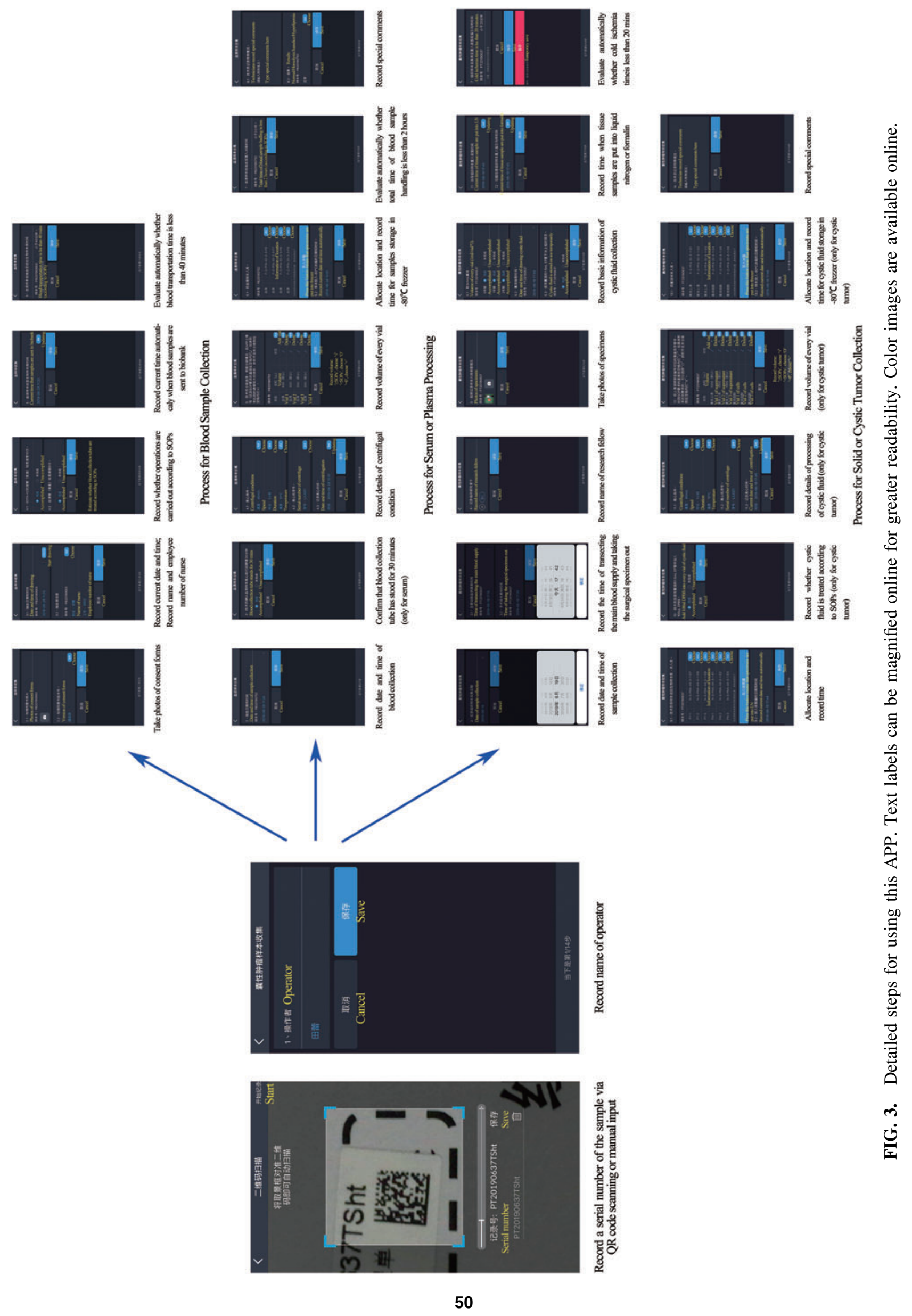


(11) For cystic tumor samples, record details of processing of cystic fluid, including preprocessing, centrifugation (speed, duration, temperature, number of device, date, and time); skip this step for solid tumor samples.

(12) Record volume of every vial; skip this step for solid tumor samples.

(13) Allocate location and record time when cystic fluid is stored; skip this step for solid tumor samples.

(14) Record special comments if necessary.

All data are then saved and uploaded to the local server.

\section{Functions of PancMoBio}

Before using PancMoBio, workflow sheets were the most common solution for recording details during sample collection and processing in our pancreas biobank. This process was not time efficient and was rather limited, and was easy to be revised. PancMoBio is not only effective and accurate, but also can record some photos when necessary, such as those of consent forms and specimens resected from patients. Thus, PancMoBio has higher information volume. For better implementation of GCP principles, PancMoBio should meet the requirements of data safety, traceability, reliability, and guarantee inviolable privacy, as well as fulfillment of QC and QA standards.

For data safety, login IDs and passwords differ according to the user. Besides, all data will be uploaded to the biobank management system through a local wireless network and backup to an internal server with a regular backup plan. Data exchange is only realized in the intranet of our hospital, rather than through the Internet. Meantime, regular data backup is also performed by the internal server.

For data traceability and reliability, it is evident that all data can be collected in a timely and synchronous manner during sample collection and processing by PancMoBio. The application is efficient and convenient. For some objective information such as date and time, revision is not allowed and, therefore, data reliability is guaranteed. In addition to all data being able to be recorded at the same time of operation, the application provides a requirement from SOPs in every step during sample collection and processing, ensuring SOP adherence of operators. For example, mixing an EDTA tube softly will be noted during blood collection.

To guarantee inviolable privacy, no identifiable personal information of donors will be recorded in PancMoBio. Only unique serial numbers generated from the biobank management system could be recorded through scanning QR codes on the labels of collection tubes or through manual inputting.

This application, PancMoBio, specifically aims to manage biospecimen collection and processing. Compared with paper documentation and LIMS software, the most widely used recording methods, it is more efficient and reliable, more convenient and accurate in recording preanalytical variables, and has a greater capability in facilitating realtime quality management and quality assurance. With all the variables recorded, the integrity of biospecimen and biobank quality management is also improved.

\section{More about PancMoBio}

This study introduces a novel tool that focuses on biobanking management. Based on smartphones or tablets, popular devices that occur frequently in our daily lives, the application is more clear, portable, and convenient. With PancMoBio developed in our pancreas biobank, data collection and protection can be guaranteed, as well as conforming to QC and QA requirements.

Although PancMoBio is a professional application specifically aimed at biospecimen collection, processing, and storage, it also has many disadvantages that cannot be solved at present. The first problem is insufficient flexibility. Routinely, one tumor tissue block is cut into three small pieces and placed into liquid nitrogen, as is the case for normal tissue. However, for various reasons, it is typical for less than six biospecimen vials to be collected in our daily work. The application cannot decrease biospecimen vials optionally in this case. The other issue is that the application cannot handle multiple specimens at the same time. It is common that multiple biospecimen collection occurs concurrently, particularly with respect to blood collection. The application can only record one by one. Thus, the working efficiency is significantly reduced.

Apart from the aforementioned problems, more functions are also required to be investigated continuously, such as temperature control management of freezers. Currently, the temperature control system is connected with a computer and mobile phone. A message is sent to a technician's mobile phone if the temperature is within normal range. If temperature control is added into our application, it will be more convenient and helpful, and perhaps offer another breakthrough in biobanking.

In conclusion, preanalytical handling of biospecimens is critical for their future application in research for diagnosis and prognosis. Preanalytical requirements are as important as analytical needs for the evaluation of clinical performance of biomarkers. Preanalytical handling should be described in detail and preanalytical variables should be considered as covariates, as are age and sex. PancMoBio is a smartphone application that was developed for the IOS platform to record the condition of preanalytical handling. It is a professional product for biobanking management with respect to implementation of GCP principles. Being convenient, reliable, efficient, and precise are its obvious advantages, as well as facilitating real-time QC and QA. It should be constantly improved and promoted worldwide, but further validation with routine utilization is still warranted.

\section{Availability}

PancMoBio is a fee-based application that is unavailable for public use at present. We intend to update the version every 2 years.

\section{Acknowledgments}

We thank Professor Jun Zhao (vice president of our hospital) and the department of science and technology of our hospital for their support. We also thank all the other staff in our Pancreas Biobank (managers, technicians, and research fellows) for their hard work and support with sample collection.

\section{Author Disclosure Statement}

No conflicting financial interests exist. 


\section{Funding Information}

This study was supported by the Innovation Capability Development Project of Jiangsu Province (No. BM2015004), the Program for Development of Innovative Research Team in the First Affiliated Hospital of NJMU, the Priority Academic Program Development of Jiangsu Higher Education Institutions (PAPD, JX10231801), and the Translational Research of Early Diagnosis and Comprehensive Treatment in Pancreatic Cancer (The Research Special Fund for Public Welfare Industry of Health, 201202007).

\section{References}

1. Ellervik C, Vaught J. Preanalytical variables affecting the integrity of human biospecimens in biobanking. Clin Chem 2015;61:914-934.

2. Lehmann S, Guadagni F, Moore H, et al. Standard preanalytical coding for biospecimens: Review and implementation of the sample pre-analytical code (SPREC). Biopreserv Biobank 2012;10:366-374.

3. Bhatt A. International Council for Harmonisation E6(R2) addendum: Challenges of implementation. Perspect Clin Res 2017;8:162-166.

4. Pieterse H, Diamant Z. Good clinical practice in clinical interventional studies. Eur Clin Respir J 2014;1:1-7.

5. Pew Research Center. Smartphone ownership and internet usage continues to climb in emerging economies; 2016. www .pewglobal.org/2016/02/22/smartphone-ownership-andinternet-usage-continues-to-climb-in-emerging-economies/ (accessed October 12, 2018).

6. McKay FH, Cheng C, Wright A, et al. Evaluating mobile phone applications for health behaviour change: A systematic review. J Telemed Telecare 2018;24:22-30.

7. Vollmer Dahlke D, Fair K, Hong YA, et al. Apps seeking theories: Results of a study on the use of health behavior change theories in cancer survivorship mobile apps. JMIR Mhealth Uhealth 2015;3:e31.

8. Grundy QH, Wang Z, Bero LA. Challenges in assessing mobile health app quality: A systematic review of prevalent and innovative methods. Am J Prev Med 2016;51:10511059 .
9. Kratzke C, Cox C. Smartphone technology and apps: Rapidly changing health promotion. International Electronic $\mathrm{J}$ Health Educ 2012;15:72-82.

10. Ramirez V, Johnson E, Gonzalez C, et al. Assessing the use of mobile health technology by patients: An observational study in primarycare clinics. JMIR Mhealth Uhealth 2016;4:e41.

11. Goyal S, Nunn CA, Rotondi M, et al. A mobile app for the selfmanagement of type 1 diabetes among adolescents: A randomized controlled trial. JMIR Mhealth Uhealth 2017:5:e82.

12. Rodin A, Shachak A, Miller A, et al. Mobile apps for eye care in Canada: An analysis of the iTunes store. JMIR Mhealth Uhealth 2017;5:e84.

13. Megan ER, Elroy JA, Rebecca LW, et al. eHealth technologies to support nutrition and physical activity behaviors in diabetes self-management. Diabetes Metab Syndr Obes 2016;9:381-390.

14. Carey E, Payne KF, Ahmed N, et al. The benefit of the smartphone in oral and maxillofacial surgery: Smartphone use among maxillofacial surgery trainees and iPhone apps for the maxillofacial surgeon. J Maxillofac Oral Surg 2015;14:1-7.

Address correspondence to: Yi Miao, MD, PhD, FACS, FRCS, FICS(Hon) Pancreas Center The First Affiliated Hospital of Nanjing Medical University 300 Guangzhou Road Nanjing 210029 P.R. China

E-mail: miaoyi@njmu.edu.cn

Zipeng Lu, MD, PhD, MRCSEd Pancreas Center The First Affiliated Hospital of Nanjing Medical University 300 Guangzhou Road Nanjing 210029 P.R. China

E-mail: surgeonmark@hotmail.com 